\title{
Análisis de satisfacción en varones que presentaban cáncer de próstata localizado tratados con prostatectomía radical o radioterapia: Aspectos Psicológicos y Sociales
}

\author{
Gugliotta $\mathrm{A}^{*}, * *$ Ferreira U**, Reis LO**, Matheus WE**, Denardi F**, Mamprim Stopiglia R, \\ Piccolotto Naccarato AME**. \\ *División de Urología, Universidad Católica Pontificia de Campinas - PUC Campinas, São Paulo, Brazil. \\ **División de Urología, Universidad Estatal de Campinas - Unicamp, São Paulo, Brazil.
}

Actas Urol Esp. 2008;32(4):411-416

\section{RESUMEN}

ANÁLISIS DE SATISFACCIÓN EN VARONES QUE PRESENTABAN CÁNCER DE PRÓSTATA LOCALIZADO TRATADOS CON PROSTATECTOMÍA RADICAL O RADIOTERAPIA: ASPECTOS PSICOLÓGICOS Y SOCIALES

Objetivo: Analizar la satisfacción y el impacto del tratamiento en pacientes con cáncer de próstata localizado.

Métodos: 180 pacientes con una edad media de 60 años que fueron divididos en 3 grupos: grupo I - 100 pacientes que se habían sometido a prostatectomía radical retropúbica (PRR), grupo II- 40 pacientes sometidos a radioterapia (RT), y grupo III - 40 hombres sanos. Se aplicó un cuestionario a los grupos para valorar los cambios físicos y psicológicos a los 18 meses tras el tratamiento. La herramienta de investigación se basó en dos cuestionarios; primero: SF-36 (Short Form Health Survey), segundo: FACT-P (Functional Assessment Cancer Therapy).

Resultados: En el grupo I, el 70\% nunca usó compresas, el 5\% tuvo incontinencia urinaria total, y el 10\% pérdidas ocasionales de heces. En el grupo II, 85\% no utilizó compresas y el 5\% informó haber utilizado dos compresas al día; 15\% reportaron incontinencia fecal. La disfunción sexual fue similar en ambos grupos: $75 \%$ del grupo de cirugía y el 72,5\% del de radioterapia informaron de disfunción eréctil. En el grupo de control, 40\% informaron de disfunción eréctil; $10 \%$ pérdidas ocasionales de heces y ninguno tuvo cambios por lo que respecta a la satisfacción global con el tratamiento. Setenta y ocho por ciento del grupo de PRR y el $77,5 \%$ del de RT informaron sentirse felices y satisfechos del tratamiento aceptado o elegido y afirmaron que lo elegirían de nuevo.

Conclusiones: La valoración de la satisfacción relacionada con el tratamiento determina la tolerancia al mismo. Los resultados de este estudio no muestran cambios importantes en este aspecto entre ambas modalidades de tratamiento $(\mathrm{p}>0,05)$.

Palabras clave: Cáncer de próstata. Prostatectomía radical. Radioterapia. Aspectos psicológicos y sociales. Calidad de vida.

\section{ABSTRACT}

SATISFACTION ANALYSIS IN MEN PRESENTING WITH LOCALIZED PROSTATE CANCER TREATED WITH RADICAL PROSTATECTOMY OR RADIOTHERAPY: PSYCHOLOGICAL AND SOCIAL ASPECTS

Objective: Analyze the treatment satisfaction and impact on patients with localized prostate cancer.

Methods: One-hundred and eighty patients, with mean age of 60 years, were divided into three groups: group I - 100 patients submitted to radical retropubic prostatectomy (RRP), group II - 40 patients who underwent radiotherapy (RT), and group III - 40 healthy men. A questionnaire was applied to the groups to assess physical and psychological changes 18 months after treatment. The investigational tool was based on two questionnaires; first: SF-36 (Short Form Health Survey), second: FACT-P (Functional Assessment Cancer Therapy).

Results: In group I, 70\% never used pads, 5\% presented with complete urinary incontinence, and 10\% reported occasional stool leakage. In group II, $85 \%$ did not use pads and 5\% reported two pads a day; 15\% reported stool leakage or intestinal cramps. Sexual dysfunction was similar in both groups: $75 \%$ of the surgical group and $72.5 \%$ of the radiotherapy group reported erectile dysfunction. In the control group, $40 \%$ reported erectile dysfunction; 10\% reported occasional stool leakage and none had changes regarding the overall treatment-related satisfaction. Seventy-eight percent of the RRP group and $77.5 \%$ of the RT group reported being happy respecting satisfaction with the accepted or chosen treatment, and affirmed that would choose it again. Conclusions: The assessment of treatment-related satisfaction determines the treatment tolerability. This study's results did not show any significant changes in this issue between both treatment modalities $(\mathrm{p}>0.05)$.

Keywords: Prostate cancer. Radical prostatectomy. Radiotherapy. Psychological and social aspects. Quality of life. 
$\mathrm{C}$ on el fin de mejorar los resultados del tratamiento del cáncer de próstata localizado, la investigación ha evolucionado básicamente en dos frentes: en primer lugar: mejorar los conocimientos sobre la historia natural de la neoplasia y su epidemiología, y segundo: entender las consecuencias del tratamiento en la satisfacción y el estilo de vida de los pacientes.

El término "satisfacción" está relacionado con la evaluación del paciente respecto a las consecuencias derivadas del tratamiento y su impacto en el estilo de vida.

Es bien sabido que los datos de supervivencia, los índices de complicaciones y la respuesta terapéutica no proporcionan información suficiente para evaluar la satisfacción del paciente. Una adecuada evaluación de la satisfacción del paciente debería tener en cuenta aspectos físicos, sexuales y condiciones psicológicas antes y después del tratamiento. A pesar de que la valoración clínica del médico no siempre expresa el sentimiento real del paciente, puede reproducir su satisfacción relacionada con el tratamiento ${ }^{1}$.

Ninguna de las opciones indicadas para el tratamiento de cáncer de próstata localizado mostró una diferencia significativa en la supervivencia, por el contrario la prostatectomía radical y la radioterapia pueden causar complicaciones importantes, dando lugar a alteraciones en el estilo de vida de los pacientes. La evaluación de los resultados del tratamiento debe ser multidimensional con una perfecta comprensión de la relación entre la repercusión física y psicosocial de cada paciente ${ }^{3-7}$.

\section{MATERIALES Y MÉTODOS}

En el presente estudio participaron 180 pacientes con edades comprendidas entre los 47-75 años (edad media de 60 años), tratados por cáncer de próstata localizado en nuestro centro, y 40 hombres sanos dentro de un rango de edad similar, pero sin cáncer de próstata (grupo de control). Se dividieron en tres grupos: grupo I, incluía 100 pacientes que se sometieron a PRR, grupo II 40 pacientes sometidos a RT conformacional, y el grupo de control compuesto por 40 pacientes.

Los criterios para seleccionar los sujetos a participar en el grupo de estudio fueron los siguientes: 1) pacientes con cáncer de próstata localizado (T1/T2); 2) firmar un consentimiento por escrito; 3) cumplir dos criterios de inclusión: a) ausencia de síntomas clínicos de fracaso terapéutico o de progresión de la enfermedad, b) niveles indetectables (o por debajo de 0,4 $\mathrm{ng} / \mathrm{ml}$ ) de antígeno prostático específico (PSA) para el grupo de cirugía, y por debajo de $0,7 \mathrm{ng} / \mathrm{ml}$ PSA nadir para el grupo II, sin subidas consecutivas.

Todos los pacientes en el grupo de cirugía se sometieron a PRR, mediante la técnica descrita ampliamente en el literatura ${ }^{8,9}$. Se preservaron los haces neurovasculares, cuando fue posible.

Todos los pacientes en el grupo de radioterapia (RT) recibieron dosis de hasta 7500 cGy, basados en la valoración del volumen tumoral mediante TAC y posterior radioterapia conformacional. Ningún paciente recibió terapia hormonal adyuvante.

A los 18 meses después del tratamiento se realizó una investigación con una muestra representativa de los grupos, con el fin de evaluar la satisfacción con respecto al tratamiento de cada paciente. La herramienta de investigación se basó en dos cuestionarios: en primer lugar: SF-36 (Short Form Health Survey), para la evaluación funcional del tratamiento del cáncer, un sondeo físico post tratamiento (limitaciones en el día a día, vitalidad, estado general y actividad social) , y un estudio psicológico (ansiedad, depresión, angustia y confusión mental); segundo: FACT-P (Evaluación Funcional Terapia del Cáncer) - un cuestionario general para evaluar los efectos secundarios tras el tratamiento (incontinencia urinaria, disfunción eréctil y toxicidad intestinal). Además, se formuló una pregunta relativa a si el paciente se sometería de nuevo al mismo tratamiento.

Estos cuestionarios proporcionaron información general acerca de las consecuencias físicas y psicológicas del tratamiento en cada paciente. La evaluación de la incontinencia urinaria se midió por la declaración y la frecuencia de cambios de compresa, fugas de orina, y el nivel subjetivo de malestar causado por esta complicación. Asimismo, un cuestionario similar fue utilizado para evaluar el nivel de sintomas intestinales en los tres grupos.

Tres investigadores y un psicólogo realizaron las entrevistas, intentando evitar el sesgo y la posible influencia en las respuestas. En cuanto a la disfunción sexual, los pacientes fueron evaluados sobre las erecciones espontáneas usando las preguntas del índice internacional de la disfunción eréctil (IIED). 
El chi-cuadrado y la prueba exacta de Fisher se aplicó para el análisis estadístico, y el nivel de significación establecido fue del 5\% (p <0,05).

\section{RESULTADOS}

Los datos demográficos del paciente, PSA, Gleason y estadio pretratamiento se enumeran en la Tabla 1.

En el grupo I, la incontinencia urinaria estuvo presente en el 5\% después de un periodo postoperatorio de 18 meses. Por otra parte, el 70\% de los pacientes estaban continentes (sin compresa) y el $15 \%$ requerían una compresa al día.

En el grupo de RT, el 85\% referían estar continentes, el $10 \%$ precisaban una compresa y un $5 \%$ dos compresas al día; ninguno presentó incontinencia urinaria durante el mismo periodo de seguimiento (Tabla 2).

En el grupo control, el 15\% de los pacientes mencionó leve incontinencia urinaria (fugas de orina una vez al día) y no existe una significación estadística entre ellos ( $p>0,05)$. Sólo 5 (16,7\%) de los pacientes con incontinencia (grupo I) refiere una molestia importante. En el grupo II, nadie informa de un gran malestar debido a incontinencia urinaria (Tabla 3).
Tabla 2: ¿Con qué frecuencia necesita cambiar de compresa debido a las pérdidas de orina?

\begin{tabular}{lccc}
\hline & $\begin{array}{c}\text { Cirugía } \\
\text { n (\%) }\end{array}$ & $\begin{array}{c}\text { Radioterapia } \\
\text { n (\%) }\end{array}$ & $\begin{array}{c}\text { Control* } \\
\text { n (\%) }\end{array}$ \\
\hline Nunca & $70(70)$ & $34(85)$ & $34(85)$ \\
Una vez al día & $15(15)$ & $4(10)$ & $6(15)$ \\
Dos veces al día & $10(10)$ & $2(5)$ & 0 \\
Siempre & $5(5)$ & 0 & 0 \\
Total & 100 & 40 & 40 \\
\hline
\end{tabular}

*Valor de $\mathrm{p}>0,05$

Tabla 3: ¿Qué tipo de molestia le causa la incontinencia urinaria? (entre pacientes que utilizan compresas)

\begin{tabular}{lcc}
\hline & $\begin{array}{c}\text { Cirugía } \\
\text { n (\%) }\end{array}$ & $\begin{array}{c}\text { Radioterapia* } \\
\text { n (\%) }\end{array}$ \\
\hline Ninguna & $20(66,6)$ & $5(83,3)$ \\
Poca & $5(16,7)$ & $1(16,7)$ \\
Mucha & $5(16,7)$ & 0 \\
Total & 3 & 6 \\
\hline
\end{tabular}

*Valor de $\mathrm{p}>0,05$

En el grupo I $75 \%$ y $72,5 \%$ en el grupo II (p> $0,05)$ comunicaron ausencia de erección en relación a la función sexual, sin embargo, el $40 \%$ del grupo de control también presentaba disfunción eréctil.

En el grupo I, sólo 20 pacientes (20\%) respondió a fármacos orales o inyecciones intracavernosas, hecho que no objetivo en el grupo II (Tabla 4).

Tabla 1: Características de los grupos entrevistados

\begin{tabular}{|c|c|c|c|c|}
\hline \multicolumn{2}{|l|}{ Grupos } & Cirugia & Radioterapia & Control \\
\hline \multicolumn{2}{|c|}{ Rango edad (Edad media) } & $47-75(60)$ & $47-75(70)$ & $40-75(58)$ \\
\hline \multirow[t]{4}{*}{ Educación } & Analfabeto & $4(4 \%)$ & $3(7,5 \%)$ & 0 \\
\hline & Elemental & $40(40 \%)$ & $10(25 \%)$ & $16(40 \%)$ \\
\hline & Enseñanza Secundaria & $44(44 \%)$ & $25(62,5 \%)$ & $20(50 \%$ \\
\hline & Enseñanza Superior & $12(12 \%)$ & $2(5 \%)$ & $4(10 \%)$ \\
\hline \multirow[t]{3}{*}{ Raza } & Caucasiano & $55(55 \%)$ & $25(62,5 \%)$ & $20(50 \%)$ \\
\hline & Descendiente Africano & $25(25 \%)$ & $13(32,5 \%)$ & $16(40 \%)$ \\
\hline & Mulato & $20(20 \%)$ & $2(5 \%)$ & $4(10 \%)$ \\
\hline \multirow[t]{4}{*}{ Ocupación } & Trabajador & $30(30 \%)$ & $8(20 \%)$ & $4(10 \%)$ \\
\hline & Jubilado & $40(40 \%)$ & $27(67,5 \%)$ & $24(60 \%)$ \\
\hline & Desempleado & $22(22 \%)$ & $5(12,5 \%)$ & $12(30 \%)$ \\
\hline & De baja & $8(8 \%)$ & 0 & 0 \\
\hline \multirow[t]{3}{*}{ Estado Civil } & Casado & $60(60 \%)$ & $30(75 \%)$ & $24(60 \%)$ \\
\hline & Viudo & $30(30 \%)$ & $5(12,5 \%)$ & $10(25 \%)$ \\
\hline & Divorciado & $10(10 \%)$ & $5(12,5 \%)$ & $6(15 \%)$ \\
\hline \multicolumn{2}{|c|}{ PSA pretratamiento } & $4-12$ & $5-10$ & \\
\hline \multicolumn{2}{|c|}{ Gleason pretratamiento } & $4-8$ & $4-7$ & \\
\hline Estádio & $\mathrm{Tlc}$ & $48(48 \%)$ & $21(50 \%)$ & \\
\hline \multirow[t]{3}{*}{ Pretatamiento } & $\mathrm{T} 2 \mathrm{a}$ & $30(30 \%)$ & $8(20 \%)$ & \\
\hline & $\mathrm{T} 2 \mathrm{~b}$ & $22(22 \%)$ & $12(30 \%)$ & \\
\hline & Total (n) & 100 & 40 & 40 \\
\hline
\end{tabular}


Tabla 4: Valoración de dos aspectos de la IQED de la disfunción eréctil: 18 meses después de la PRR

\section{¿Con qué frecuencia puede tener erecciones durante la actividad sexual?}

$\begin{array}{lc}\text { Cirugia }(\mathbf{n}=\mathbf{1 0 0}) & \text { Radioterapia }(\mathbf{n = 4 0}) \\ 0-1=75(75 \%)^{*} & 0-1=29(72,5 \%)^{*} \\ 2=15(15 \%) & 2=0 \\ 3-5=5(5 \%) & 3-5=11(27,5 \%)\end{array}$

Control (n=40)

$0-1=17(40 \%)$

$2=3(7,5 \%)$

$3-5=20(50 \%)$

¿Cuándo tiene erecciones, con qué frecuencia puede penetrar?

$\begin{array}{lc}0-1=75(75 \%)^{*} & 0-1=29(72,5 \%)^{*} \\ 2=15(15 \%) & 2=0 \\ 3-5=5(5 \%) & 3-5=11(27,5 \%)\end{array}$

Escala del 1 al 5 (IQED): 0 = sexualmente inactivo; 1 = raramente/nunca; 2 = poco; 3 = parcialmente; 4 = la mayoría de las veces; $5=$ siempre.

En el grupo II, seis pacientes (15\%) informaron de incontinencia fecal leve, de los cuales cuatro pacientes (10\%) presentaron de episodios de rectorragia. En el grupo I, estos síntomas fueron del 10\% (incontinencia fecal leve), $\mathrm{p}>0,05$.

Con relación a la satisfacción con el tratamiento aceptado o elegido, el 78\% del grupo de PRR y el $77,5 \%$ del de RT informaron que estaban contentos y que escogerían el mismo si tuvieran una segunda oportunidad ( $\mathrm{p}>0,05)$ (Tabla 5).

\section{DISCUSIÓN}

Los hombres que presentaron cáncer de próstata localizado (T1/T2) recibieron orientación de por lo menos una de las tres modalidades de tratamiento a seguir: PRR, RT o la observación clínica.

En este estudio, la tasa de pacientes totalmente continentes fue del 70\%, similar a la que muestran otros estudios ${ }^{2,7,15}$.

Se pueden asignar varios niveles de incontinencia urinaria que han sido comunicados en la literatura pero no existe uniformidad en la definición de la incontinencia urinaria y el método de evaluación.
Algunos cirujanos dicen que la incontinencia tras PRR es tiempo-dependiente ${ }^{16-18}$. En el presente estudio, los pacientes fueron identificados como continentes si nunca habían usado compresas, incluida la incontinencia de esfuerzo (sin uso previo de compresas). Esto aumenta la tasa total de la evaluación. Por ejemplo, si consideramos significativo utilizando una compresa al día, nuestra tasa de incontinencia en el estudio actual aumentaría del $15 \%$ al 30\%, similar a la existente en los estudios disponibles $^{7,18}$.

Con respecto a la edad, parece que los pacientes de más de 70 años no recuperan muy fácilmente la continencia. Otros estudios han informado de que la edad tendría un impacto menor, ya que algunos casos fueron seleccionados para cirugía en este grupo de edad $8,19,20$.

Aunque los pacientes de RT refieren menos incontinencia y algunos de ellos tuvieron que usar compresas (15\%), el índice de incomodidad fue similar entre los grupos. Por otro lado, un número considerable de pacientes que se habían sometido a RT tuvieron importantes síntomas del tracto urinario inferior.

Tabla 5: Valoración de la calidad de vida (*) de los grupos respecto a los aspectos emocionales, psicológicos y sociales

\begin{tabular}{|c|c|c|c|c|c|c|}
\hline & Valor de $p$ & $\begin{array}{c}\text { Cirugía } \\
\text { n (\%) }\end{array}$ & Valor de $p$ & $\begin{array}{c}\text { Radioterapia } \\
\text { n (\%) }\end{array}$ & Valor de $p$ & $\begin{array}{c}\text { Contro } \\
\text { n (\%) }\end{array}$ \\
\hline Depresión & 3 & $70(70)$ & 3 & $15(37,5)$ & 2 & $34(85)$ \\
\hline Ira & 3 & $70(70)$ & 3 & $13(32,5)$ & 2 & $34(85)$ \\
\hline Estrés & 3 & $64(64)$ & 3 & $27(67,5)$ & 3 & $36(90)$ \\
\hline Fatiga & 2 & 45 (45) & 4 & $27(67,5)$ & 3 & $24(60)$ \\
\hline Confusión & 2 & $53(53)$ & 3 & $27(67,5)$ & 3 & $16(40)$ \\
\hline Vida social & 3 & $45(45)$ & 4 & $28(70)$ & 4 & $8(20)$ \\
\hline Bienestar físico & 3 & $60(60)$ & 3 & $27(67,5)$ & 3 & $16(40)$ \\
\hline Total & & 100 & & 40 & & 40 \\
\hline
\end{tabular}

*Puntuación de 0 a 5, siendo el número mayor el peor índice de calidad de vida. 
Tabla 6: Probabilidad de que el paciente siguiera el mismo tratamiento, si tuviera una segunda oportunidad para escoger

\begin{tabular}{lcc}
\hline & $\begin{array}{c}\text { Prostatectomia } \\
\text { radical } \\
\text { n (\%) }\end{array}$ & $\begin{array}{c}\text { Radioterapia* } \\
\text { n (\%) }\end{array}$ \\
\hline Definitivamente Si & $62(62)$ & $18(45)$ \\
Probablemente Si & $16(16)$ & $13(32,5)$ \\
Probablemente No & $20(20)$ & $6(15)$ \\
Definitivamente No & $2(2)$ & $3(7,5)$ \\
Total & 40 & 100 \\
\hline
\end{tabular}

*Valor de $\mathrm{p}>0,05$

La disfunción eréctil afecta a entre el 10\% y el 90\% de los hombres después de PRR y del 2\% al $34 \%$ de los hombres sometidos a RT, es decir, menos de lo que pudimos observar en este estudio $^{21,25}$. También es importante mencionar que en el grupo de control un $40 \%$ de los pacientes presentaban cierto grado de disfunción eréctil.

El actual estudio no mostró diferencias importantes entre los grupos de RT y PRRVP (75\% frente a $72,5 \%$ ) con respecto a la prevalencia de disfunción eréctil.

Según otros estudios similares ${ }^{7,15}$, el análisis de satisfacción del paciente relacionado con el tratamiento fue expresada en ambos grupos, y no se informó de ninguna diferencia significativa entre los grupos de PRR y RT (p>0,05).

\section{CONCLUSIÓN}

Los resultados del análisis no mostraron diferencias significativas entre los grupos con relación a los aspectos psicológicos y sociales. La incontinencia urinaria fue frecuente en el grupo I, y los problemas intestinales en el grupo II; sin embargo no existió diferencia estadística. La disfunción eréctil fue similar en ambos grupos. Asimismo, la probabilidad de que un paciente se sometiera de nuevo al mismo tratamiento no fue estadísticamente significativa, entre ambas opciones.

En conclusión la satisfacción puede medirse bien de acuerdo con las respuestas y quejas de los pacientes. A pesar de que sean casi similares, hay diferencias entre los grupos en cuanto a las complicaciones. Sin embargo, en general ambos modalidades de tratamiento ofrecen buena aceptación y satisfacción.
Estos hallazgos pueden proporcionar información importante para ayudar a pacientes y urólogos a escoger el tratamiento más adecuado para el cáncer localizado de próstata.

\section{REFERENCIAS}

1. Macdonagh R. Quality of life and its assessments in urology. Br.J.Urol. 1996;78(4):485-496.

2. Litwin MS, Hays RD, Fink A, Ganz PA, Leake B, Leach GE, et al. Qualiy of life outcomes in men treated for localized prostate cancer. JAMA. 1995;273(2):129-135.

3. Fosså SD, Woehre H, Kurth KH, Hetherington J, Bakke H, Rustad DA, et al. Influence of urological morbidity on quality of life in patients with prostate cancer. Eur Urol. 1997;31 Suppl 3:3-8.

4. Krongrad A, Litwin MS, Lai H, Lai S. Dimensions of quality of life in prostate cancer. J.Urol. 1998;160(3 Pt 1):807-810.

5. Litwin MS, Lubeck DP, Henning JM, Flanders SC. Differences in urologist and patient assessments of health related quality of life in men with prostate cancer. J Urol. 1998;159(6):19881991.

6. Clark JA, Rieker P, Propert JK, Talcott JA. Changes in quality of life following treatment for early prostate cancer. Urology. 1999;53(1):161-168.

7. McCammon KA, Kolm P, Main B, Schelhammer PF. Comparative quality of life analysis after radical prostatectomy or external beam radiation for localized prostate cancer. Urology. 1999;54(3):509-516.

8. Walsh PC, Lepor H, Eggleston JC. Radical retropubic prostatectomy with preservation of sexual function: anatomical and pathological considerations. Prostate. 1983;4(5):473-476.

9. Walsh PC. Radical prostatectomy for localized prostate cancer provides durable cancer control with excellent quality of life: A structured debate. J Urol. 2000;163(6):1166-1170.

10. Caffo O, Fellini G, Graffer U, Luciani L. Assessment of quality of life after radiotherapy. B J Urol. 1996;78(4):557-563.

11. Esper P, Mo F, Chodak G, Sinner M, Cella D, Pienta KJ. Measuring quality of life in men with prostate cancer using the Functional Assesment of Cancer Therapy-Prostate Instrument. Urology. 1997;50(6):920-928.

12. Krongrad A, Perczek RE, Burke MA, Granville LJ, Lai H, Lai S. Reliability of spanish translations of select urological quality of life instruments. J Urol. 1997;158(2):493-498.

13. Brasilis KG, Santa-Cruz C, Brickman AL, Soloway MS. Quality of life 12 months after radical prostatectomy. B J Urol. 1995;75 (1):48-53.

14. Smith DS, Carvalhal GF, Schneider K, Krygiel J, Yan Y, Catalona WJ. Quality of life outcomes for men with prostate cancer detected by screening. Cancer. 2000;88(6):1454-1463.

15. Lim AJ, Brandon AH, Fiedler J, Brickman AL, Boyer CI, Raub WA Jr, Q Quality of life : Radical Prostatectomy versus Radiation Therapy for prostate cancer. JAMA. 1998;272:129-135.

16. Goluboff ET, Saidi JA, Mazer S, Bagiella E, Heitjan DF, Benson $\mathrm{MC}$, et al. Urinary incontinence after radical prostatectomy: The Columbia Experience. J Urol. 1998;159(4):1276-1280.

17. Kaye KW, Creed KE, Wilson GJ, D’Antuono M, Dawkins HJ. Urinary continence after radical retropubic prostatectomy. Analysis and synthesis of contributing factors: a unified concept. B J Urol. 1997;80(3):444-501.

18. Twiss C, Martin S, Shore R, Lepor H. A continence index predicts the early return of urinary continence after radical retropubic prostatectomy. J Urol. 2000;164(4):1241-1247. 
19. Kerr LA, Zincke H. Radical retropubic prostatectomy for prostate cancer in the elderly and young: complications and prognosis. Eur Urol. 1994;25(4):305-311.

20. Beard CJ, Propert KJ, Rieker PP, Clark JA, Kaplan I, Kantoff $\mathrm{PW}$, et al. Complications after treatment with external-beam irradiation in early-stage prostate cancer patients: a prospective multiinstitutional outcomes study. J Clin Oncol. 1997;15(1): 223-229.

21. Geary ES, Dendinger TE, Freiha ES. Nerve sparing radical prostatectomy: a different view. J Urol. 1995;154(1):145-149.

22. Litwin MS, Pasta DJ, Yu J, Stoddard ML, Flanders SC. Urinary function and bother after radical prostatectomy or radiation therapy for prostate cancer. A longitudinal, multivariate quality of life analysis from the Cancer of Prostate Strategic Urologic Research Endeavor. J Urol. 2000;164(6):1973-1977.

23. Zelefsky MJ, McKee AB, Lee H, Leibel SA. Efficacy of oral sildenafil in patients with erectile dysfunction after radiotherapy for carcinoma of the prostate. Urology. 1999;53(4):775-778.

24. Johannes CB, Araujo AB, Feldman HA, Derby CA, Kleinman $\mathrm{KP}$, McKinlay JB. Incidence on erectile dysfunction in men 40 to 69 years old: longitudinal results from the Massachusetts Male Aging Study. J Urol. 2000;163(2):460-465.
25. Siegel T, Moul JW, Sperak M, Alvord GW, Costabile RA. The development of erectile dysfunction in men treated for prostate cancer. J Urol. 2001;165(2):430-435.

26. Gralnek D, Hunter W, Dalkin BL. Differences in sexual function and quality of life after nerve sparing and non nerve sparing radical retropubic prostatectomy. J Urol. 2000;163:11661170 .

27. Nehra A, Goldstein I. Sildenafil citrate (Viagra) after radical retropubic prostatectomy: Con Urology. 1999;54(4):587-589.

Correspondencia autor: Dr. Leonardo Oliveira Reis División de Urologia. Universidad Estatal de Campinas Unicamp, São Paulo. Brasil

R. Votorantim, 51, ap. 43, Campinas-SP, Brasil - 13073-090 Tel.:/Fax: + $55193521-7481$

E-mail autor: reisleo@unicamp.br

Información artículo: Original - Cáncer de próstata

Trabajo recibido: septiembre 2007

Trabajo aceptado: diciembre 2007 